\title{
Percepción de los estudiantes de enfermería sobre sus conocimientos acerca de la enfermería nefrológica
}

\author{
Beatriz Sánchez-Pérez ${ }^{1}$, Carmen Guerra-Cuetoํㅜㄹ ${ }^{1}$ Miguel Núñez-Moral ${ }^{1,2,3}$
}

1 Unidad de Gestión Clínica Nefrología. Hospital Universitario Central de Asturias. Oviedo. España

2 Departamento de Medicina, Área de Enfermería. Universidad de Oviedo. Oviedo. España

3 Grupo de Investigación en Cuidados. Instituto de Investigación Sanitaria del Principado de Asturias (ISPA). Oviedo. España

Como citar este artículo:

Sánchez-Pérez B, Guerra-Cueto C, Núñez-Moral B. Percepción de los estudiantes de enfermería sobre sus conocimientos acerca de la enfermería nefrológica.

Enferm Nefrol. 2020 0ct-Dic;23(4):389-395

\section{Resumen}

Introducción: La enfermedad renal crónica avanzada supone un gran problema sanitario para el que son primordiales los cuidados de enfermería y por tanto la formación de los futuros profesionales.

Objetivo: evaluar la percepción de conocimientos y el grado de interés en enfermería nefrológica de los estudiantes de enfermería de la Universidad de Oviedo.

Material y Método: Estudio observacional descriptivo desarrollado entre enero-mayo de 2019, se incluyeron todos los alumnos matriculados del grado de enfermería que dieron su consentimiento por escrito. Se utilizó un cuestionario realizado por los investigadores, que incluyo variables sociodemográficas y 12 preguntas cerradas, formadas por dos tipos de respuesta: dicotómicas (si/no/ no sabe) y tipo Likert (escala de 0 a 10). Se realizó un análisis descriptivo y se utilizaron las pruebas $T$ de Student y ANOVA para buscar diferencias significativas. Resultados: Obtuvimos 251 cuestionarios, con un índice de rechazo $<1 \%$. El $86 \%$ eran de mujeres entre 18-54 años. La media de conocimiento total para los cuatro cursos fue $<5$, habiendo encontrado diferencias estadísticamente significativas por edad $(p<0,003)$, pero

\section{Correspondencia:}

Carmen Guerra Cueto

Email: carmencitagc23@gmail.com no por sexo, curso o profesión. La importancia de la

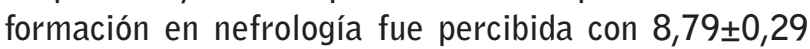
puntos de media y el interés por formarse en enfermería nefrología es de 3,82 $\pm 0,61$ puntos de media.

Conclusiones: La percepción de conocimientos de los alumnos en enfermería nefrológica es insuficiente a pesar de percibirlo como muy necesarios para su formación, además, en caso de que existiera, presentan escaso interés en realizar la especialidad.

PALABRAS CLAVE: enfermería en nefrología; enfermedad renal crónica; estudiantes de enfermería; conocimiento; diálisis renal.

\section{Nursing students' perception of their knowledge about nephrology nursing}

\section{Abstract}

Introduction: Advanced chronic kidney disease is a major health problem in which nursing cares, and therefore the training of future professionals, are essential.

Objective: To evaluate the perception of knowledge and degree of interest in nephrology nursing of nursing students at the University of Oviedo. 
Material and Method: Descriptive observational study carried out between January and May 2019, including all the students enrolled in the nursing degree who gave written consent. A self-prepared questionnaire was used, which included sociodemographic variables and 12 closed questions with categorical responses (yes / no / don't know) and Likert type (scale from 0 to 10). A descriptive analysis was performed and Student's $t$ tests and ANOVA were used to search for significant differences.

Results: Two hundred and fifty-one questionnaires were obtained, with a rejection rate of less than $1 \% .86 \%$ were women between 18-54 years old. The average of total knowledge for the four courses was less than 5 , finding statistically significant differences by age ( $p$ $<0.003$ ), but not by sex, academic year or profession. The importance of nephrology training was perceived with a mean of $8,79 \pm 0,29$ points, and interest in nephrology nursing training had a mean of $3,82 \pm 0,61$ points.

Conclusions: The students' perception of knowledge in nephrology nursing is insufficient despite perceiving it as very necessary for their training. Although, if the specialty existed, they show little interest in this training.

KEYWORDS: nephrology nursing; chronic kidney disease; students, nursing; knowledge; renal dialysis

\section{Introducción}

La enfermedad renal crónica avanzada (ERCA) supone un problema sanitario de especial relevancia debido a su magnitud y a los elevados costes que conlleva tanto a nivel personal como sociosanitario $0^{1,2}$.

A nivel mundial se estima que 1 de cada 10 personas desarrollará enfermedad renal crónica (ERC) ${ }^{3}$. En España, la prevalencia se sitúa por encima de la media europea (13\%) habiendo aumentado hasta un 23\% en los casos más graves de ERC que requieren tratamiento sustitutivo renal (TSR). Asturias es una de las comunidades autónomas con mayor incidencia ( $>150$ personas por millón de población). Actualmente el número de pacientes sometidos a TSR, según el registro de la Sociedad Española de Nefrología, supera ya los 120 por millón de población, lo que implica a más de 57.000 personas $^{4}$.
A pesar de que los pacientes renales suponen un porcentaje pequeño de la población enferma los recursos consumidos suponen una gran parte del gasto sanitario $(2,5 \% \text { del presupuesto del sistema nacional de salud })^{5}$. En 2010 el impacto económico del TSR rondaba los 1.829 millones de euros, actualmente se encuentra en torno a 2.289 millones de euros y las previsiones para el futuro se traducen en cantidades ingentes ${ }^{6}$.

La mayoría de la población afectada por esta enfermedad se encuentra comprendida entre los 45 y 64 años, probablemente debido a que los principales factores de riesgo son hipertensión arterial, diabetes meIlitus, dislipemia, tabaquismo y obesidad ${ }^{7}$. Por grupos de edad, los mayores de 65 años representan al 50\% de la población sometida TSR. En los próximos años se estima que la población española mayor de 65 años aumentará de forma considerable hasta llegar a los 13 millones en $2036^{6}$.

La enfermera es pieza clave durante el tratamiento y cuidado del paciente renal6. Aun así, los alumnos de Grado de Enfermería no alcanzan la formación suficiente sobre el paciente nefrológico ${ }^{8}$ y no todos tienen la oportunidad de realizar prácticas clínicas en una Unidad de Nefrología9. Y no sólo el conocimiento de los estudiantes sanitarios es limitado, también el del profesional enfermero ${ }^{10}$, sobre todo si nos centramos en la modalidad de diálisis peritoneal, según Martín et al., más del $55 \%$ de los enfermeros encuestados en un servicio de nefrología no sabrían actuar frente a las complicaciones más habituales de la DP ${ }^{11}$.

Por este motivo el objetivo de nuestro estudio fue evaluar la percepción que tienen los estudiantes de la Universidad de Oviedo sobre sus conocimientos en Enfermería Nefrológica y el grado de interés por la misma.

\section{Material y Método}

Se realizó un estudio observacional descriptivo desarrollado de enero a mayo de 2019. Se realizó un muestreo no probabilístico intencional.

Se incluyó a todos los alumnos de Grado de Enfermería de la Universidad de Oviedo que cumplían con los criterios de inclusión: aceptar participar en el estudio, otorgando el consentimiento informado por escrito y estar matriculado en el Grado de Enfermería de la Universidad de Oviedo. 
Las variables a estudio fueron la edad, sexo, curso académico (alumno que está matriculado en al menos $80 \%$ de los créditos de un curso académico) y profesión (aparte de ser estudiante, si cuenta con otra profesión, y si esta es sanitaria).

Se utilizó un cuestionario realizado por los investigadores principales que está basado en la bibliografía, que incluía variables sociodemográficas y 12 preguntas cerradas, formadas por dos tipos de respuesta, tipo dicotómicas (si/no/no sabe) y tipo Likert (escala de 0 a 10 , siendo 0 conocimiento nulo y 10 alto). Una vez cumplimentados, fueron depositándolos en urnas para salvaguardar el anonimato.

Los datos fueron analizados utilizando el programa estadístico Excel $^{\circledR}$, las variables cuantitativas se describieron usando la media y desviación estándar y para las variables cualitativas se expresaron en frecuencias (absolutas y relativas). Para comprobar si existían diferencias significativas en función del sexo y en función de la edad se utilizó la prueba estadística T de Student y se llevó a cabo el análisis de varianza de un factor (ANOVA) para analizar el curso académico y la profesión. EI nivel de significación estadística se fijó en $p<0,05$.

El estudio se llevó a cabo cumpliendo la Ley Orgánica 3/2018, de 5 de diciembre de protección de datos personales y garantía de los derechos digitales. El estudio contó con el informe favorable del Comité de Ética e Investigación del Hospital Universitario Central de Asturias.

\section{Resultados}

El número de participantes en el estudio fue 251 de 285 alumnos matriculados en el Grado de Enfermería de la Universidad de Oviedo. De los 34 alumnos que no participaron, 2 declinaron formar parte del estudio y el resto no asistieron a clase el día de la cumplimentación del cuestionario. El porcentaje de cumplimentación total fue del $88 \%$ y el índice de rechazo $<1 \%$. La edad de los participantes comprendía entre 18-54 años. Siendo la media de conocimiento para los de 18 años $(2,88 \pm 2,66)$ y para los de $54(5,62 \pm 1,88)$.

fi=frecuencia absoluta $/ /$ hi= frecuencia relativa.
De los 251 estudiantes, el $86 \%$ eran mujeres $(n=216)$. La media de conocimiento total de este colectivo fue de $4,59 \pm 0,80$, frente al $4,80 \pm 1,16$ de los hombres.

Un $87 \%$ de los participantes eran estudiantes (media conocimiento total de los cuatro cursos fue de $4,54 \pm 0,85)$, mientras que un $10 \%$ eran estudiantes y profesionales sanitarios (media $5,13 \pm 0,87$ ) y un $3 \%$ otros (media 3,66 $\pm 2,54$ ) (Tablas 1 y 2).

Tras realizar las pruebas estadísticas pertinentes se ha comprobado que existe diferencia estadísticamente significativa en cuanto a la edad $(p<0,003)$ pero no en cuanto al sexo $(p=0,77)$, el curso académico $(p=0,21)$, ni la profesión $(p=0,47)$ (Tabla 3$)$.

\section{Discusión}

Los conocimientos en enfermería nefrológica son fundamentales para el cuidado del paciente renal. La especialidad de Grado en Enfermería tal y como está concebida puede no conseguir que los mismos se adquieran de manera adecuada. En nuestro estudio se pudo comprobar que la percepción que tienen los estudiantes de la Universidad de Oviedo sobre sus conocimientos en la enfermería nefrológica en general es bajo, como ya se ha descrito en estudios anteriores ${ }^{10}$.

Aunque no hay diferencia significativa en la comparación de medias, nuestros resultados muestran que los hombres perciben tener más conocimiento que las mujeres. Estudios previos tampoco encontraron diferencias de conocimiento teniendo en cuenta la variable sexo ${ }^{12}$.

Teniendo en cuenta la edad, comprobamos que los estudiantes de 18 años tienen menor nivel de conocimiento

Tabla 1. Características de la población a estudio por curso.

\begin{tabular}{|c|c|c|c|c|c|}
\hline \multirow[b]{2}{*}{$\begin{array}{l}\text { Población } \\
\text { a estudio }\end{array}$} & \multicolumn{4}{|c|}{ Curso académico } & \multirow[b]{2}{*}{ Total } \\
\hline & Primero & Segundo & Tercero & Cuarto & \\
\hline $\begin{array}{l}\text { N (encuestas recogidas/ } \\
\text { encuestas entregadas) }\end{array}$ & $86 / 86$ & $68 / 68$ & $62 / 64$ & $35 / 35$ & $251 / 253$ \\
\hline Sexo (mujer) & $\begin{array}{c}f i=69 \\
h i=0,27\end{array}$ & $\begin{array}{c}f i=60 \\
h i=0,23\end{array}$ & $\begin{array}{c}f i=53 \\
h i=0,21\end{array}$ & $\begin{array}{c}f i=34 \\
h i=0,13\end{array}$ & $\begin{array}{l}f i=216 \\
h i=0,86\end{array}$ \\
\hline Edad media (años) & $20,60 \pm 5,49$ & $21,88 \pm 5,26$ & $22,75 \pm 4,68$ & $24,48 \pm 6,82$ & $22,42 \pm 1,62$ \\
\hline $\begin{array}{l}\text { Profesión } \\
\text { (estudiantes) }\end{array}$ & $\begin{array}{c}f i=75 \\
h i=0,29\end{array}$ & $\begin{array}{c}f i=58 \\
h i=0,23\end{array}$ & $\begin{array}{c}f i=56 \\
h i=0,22\end{array}$ & $\begin{array}{c}f i=29 \\
h i=0,11\end{array}$ & $\begin{array}{l}f i=218 \\
h i=0,86\end{array}$ \\
\hline
\end{tabular}


que los alumnos de 54. Existe diferencia estadísticamente significativa, probablemente debido a un mayor nivel de conocimientos previos. Esto concuerda con lo publicado por Wright et $\mathrm{al}^{13}$. donde se demuestra que la formación a través de programas educativos aumenta el conocimiento sobre ERC.
En la primera pregunta, se ha visto que los alumnos creen que la prevalencia de enfermedad renal crónica en España es media, pero según la Sociedad Española de Nefrología, la prevalencia en España es baja $(13 \%)^{14}$.

Tabla 2. Resultados globales y por curso académico de los alumnos de Grado de Enfermería.

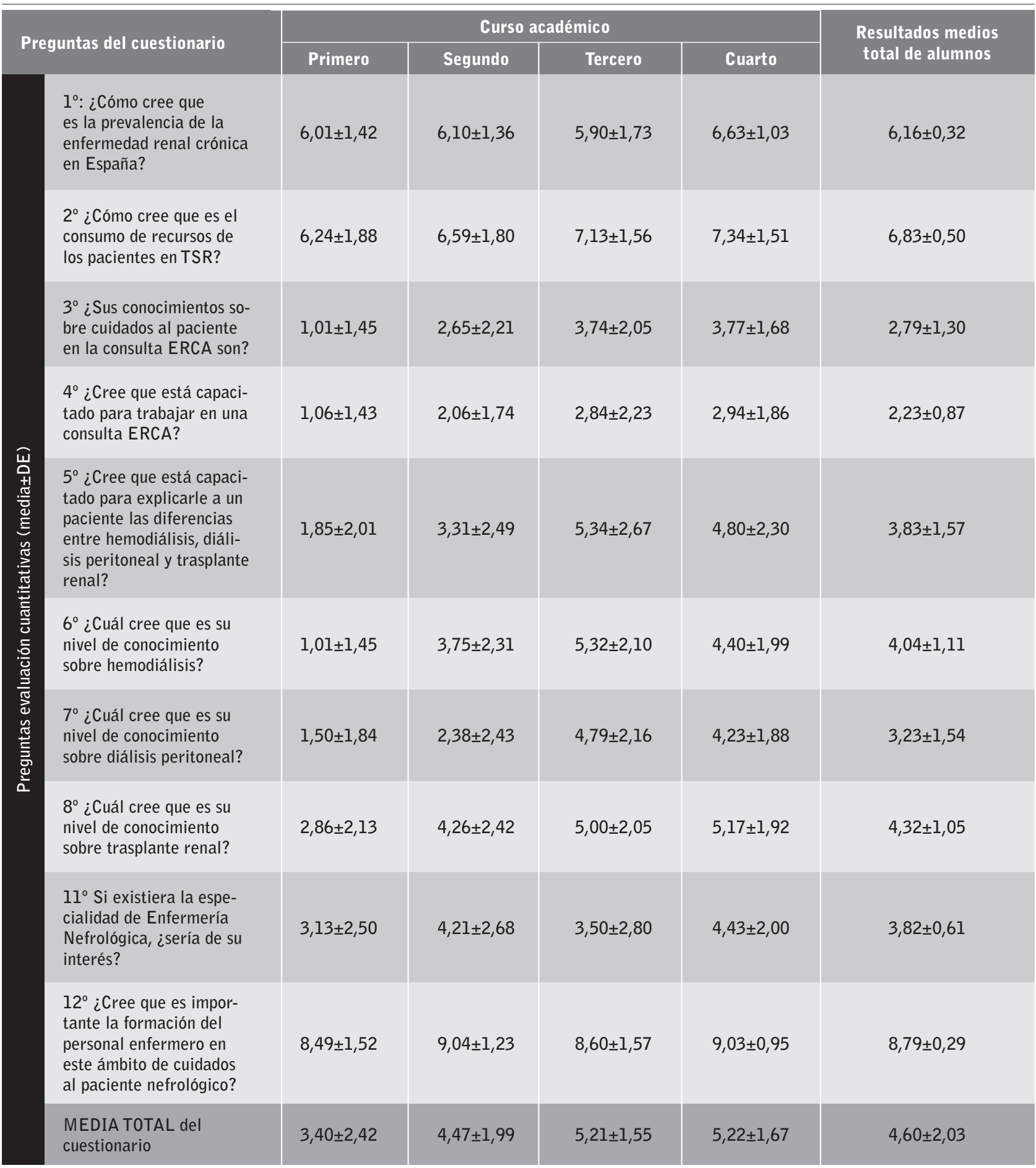




\begin{tabular}{|c|c|c|c|c|c|c|c|}
\hline \multirow{2}{*}{\multicolumn{3}{|c|}{ Preguntas del cuestionario }} & \multicolumn{4}{|c|}{ Curso académico } & \multirow{3}{*}{$\begin{array}{l}\text { Resultados medios } \\
\text { total de alumnos } \\
46 \%(n=116)\end{array}$} \\
\hline & & & Primero & Segundo & Tercero & Cuarto & \\
\hline$\frac{\pi}{0}$ & \multirow{3}{*}{$\begin{array}{l}9^{\circ} \text { A lo largo de } \\
\text { sus prácticas, ¿se } \\
\text { ha encontrado } \\
\text { con pacientes } \\
\text { sometidos a } \\
\text { TSR? }\end{array}$} & $\mathrm{Si}$ & $18 \%(n=16)$ & $49 \%(n=33)$ & $65 \%(n=40)$ & $77 \%(n=27)$ & \\
\hline$\frac{2}{20}$ & & NO & $64 \%(n=54)$ & $35 \%(n=24)$ & $35 \%(n=22)$ & $17 \%(n=6)$ & $42 \%(n=106)$ \\
\hline$\frac{\mathbb{E}}{\frac{ \pm}{\sigma}}$ & & NS & $18 \%(n=16)$ & $16 \%(n=11)$ & -- & $6 \%(n=2)$ & $12 \%(n=2)$ \\
\hline$\frac{\frac{d}{\bar{d}}}{\frac{\pi}{\sigma}}$ & \multirow{2}{*}{$\begin{array}{l}10^{\circ} \text { A lo largo } \\
\text { de sus años } \\
\text { de formación, } \\
\text { ¿alguna vez ha } \\
\text { hecho prácticas } \\
\text { en el Servicio de } \\
\text { Nefrología? }\end{array}$} & $\mathrm{Si}$ & $6 \%(n=5)$ & $15 \%(n=10)$ & $11 \%(n=7)$ & $17 \%(n=6)$ & $11 \%(n=28)$ \\
\hline 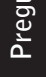 & & No & $94 \%(n=81)$ & $85 \%(n=58)$ & $89 \%(n=55)$ & $83 \%(n=29)$ & $89 \%(n=223)$ \\
\hline
\end{tabular}

Tabla 3. Puntuación media percepción de conocimiento y relación entre variables.

\begin{tabular}{|l|c|c|c|c|}
\hline \multicolumn{2}{|c|}{ Variables } & $\%$ (frecuencia) & Media \pm DE & p* \\
\hline \multirow{2}{*}{ SEX0 } & Hombre & $14 \%(37)$ & $4,80 \pm 1,16$ & 0,77 \\
\hline \multirow{2}{*}{ EDAD } & Mujer & $86 \%(216)$ & $4,59 \pm 0,80$ & \\
\hline \multirow{2}{*}{ CURSO } & 18 años & $16 \%(40)$ & $2.88 \pm 2,66$ & $<0,01$ \\
& 54 años & $0 \%(1)$ & $5,62 \pm 1,88$ & \\
& $1^{\circ}$ & $34 \%(86)$ & $3,40 \pm 2,42$ & 0,21 \\
& $2^{\circ}$ & $27 \%(68)$ & $4,47 \pm 1,99$ & \\
\hline \multirow{2}{*}{ PROFESIÓN } & $3^{\circ}$ & $25 \%(64)$ & $5,21 \pm 1,55$ & \\
& $4^{\circ}$ & $14 \%(35)$ & $5,22 \pm 1,67$ & \\
& Estudiante & $87 \%(218)$ & $4,54 \pm 0,85$ & 0,47 \\
& Sanitario & $10 \%(25)$ & $5,13 \pm 0,87$ & \\
\hline
\end{tabular}

DE: desviación estándar. * Test T de Student para comparar medias. ANOVA para comparar grupos.

El consumo de recursos de los pacientes sometidos a TSR según los alumnos es medio pero según el artículo de Arrieta J, los recursos consumidos por las personas sometidas a TSR son elevados $(2,5 \%$ del presupuesto del Sistema Nacional de Salud) ${ }^{5}$.

También se ha podido comprobar que el nivel de conocimiento de los alumnos es mayor en hemodiálisis y trasplante renal y menor en diálisis peritoneal y consulta ERCA. Esto se verifica con el estudio realizado a los profesionales de enfermería y a los médicos internos residentes ${ }^{8}$ en el cual se afirma que los cono- cimientos en este ámbito son limitados, sobre todo en la modalidad de diálisis peritoneal ${ }^{9}$.

Se ven menos capacitados para trabajar en una consulta ERCA, pero más capacitados para explicar las diferencias entre hemodiálisis, diálisis peritoneal y trasplante, pero aun así el nivel de conocimiento sigue estando lejos del aprobado.

Solo un número reducido de alumnos $(n=28)$ han tenido la oportunidad de rotar por el Servicio de Nefrología, comprobando que estos tienen mayor conocimiento que los alumnos que no han rotado por este servicio. A pesar de esto, un $46 \%$ se ha encontrado alguna vez a lo largo de sus prácticas con pacientes sometidos a alguna modalidad del TSR.

Todos los alumnos coinciden en que la formación del personal enfermero en este ámbito es muy importante, pero el interés por formarse en la especialidad de Enfermería Nefrología es bajo.

Cabe destacar la alta cumplimentación del cuestionario por los alumnos, lo que transmite su compromiso con los estudios de investigación. Pero el principal problema que nos encontramos es la baja participación de los alumnos de $4^{\circ}$ curso de Grado de Enfermería al haber una incapacidad para reunirse con los alumnos durante el periodo de recogida de datos, pero la alta cumplimentación total, nos hace interpretar que no acarrea sesgo. 
Los alumnos se podrían haber visto influidos por la presencia de los investigadores a la hora de realizar los cuestionarios, dando respuestas orientadas a representar un determinado resultado y por ello se podría haber incurrido en un sesgo del entrevistador, aunque los resultados de este estudio tienen una validación externa ya que coincide con otros realizados ${ }^{15}$.

Finalmente, los datos obtenidos en estos cuestionarios se han medido en los alumnos de la Universidad de Oviedo, sería interesante medirlo en otras universidades nacionales e internacionales para ver si los datos coinciden o es una situación académica local.

A partir de los resultados encontrados podemos afirmar que la percepción de los alumnos respecto a su nivel de conocimiento en enfermería nefrológica es escasa. Esta percepción de conocimientos es mayor en los alumnos de mayor edad. Todos los alumnos consideran que la formación del personal sanitario en cuanto a ERC y TSR es muy necesaria, aunque sólo 3 de cada 10 estudiantes estarían interesados en formar parte de un programa de especialización en este ámbito.

De nuestros datos podemos destacar la necesidad de mayor formación en enfermería nefrológica por parte de los estudiantes de enfermería de la Universidad de Oviedo.

Recepción: 5-09-20

Aceptación: 4-11-20

Publicación: 30-12-20

\section{Bibliografía}

1. Sánchez-Tomero JA. Introducción a la enfermedad renal crónica. En: La diálisis Peritoneal en la Planificación Integral del Tratamiento Sustitutivo Renal. Grupo de Apoyo al Desarrollo de la Diálisis Peritoneal en España (GADDPE). Madrid: Sociedad Española de Nefrología; 2014. p. 7-15.

2. Kramer A, Pippias M, Noordzij M, Stel VS, Afentakis N, Ambühl PM, et al. The European Renal association-European Dialysis and Transplant Association (ERA-EDTA) Registry Annual Report 2015: a summary. Clin Kidney J. 2018;11(1):108-22.
3. Bello AK, Levin A, Tonelli M, Okpechi IG, Feehally J, Harris D, et al. Global Kidney Health Atlas: A report by the International Society of Nephrology on the current state of organization and structures for kidney care across the globe. Bruselas: International Society of Nephrology; 2017.

4. Sociedad Española de Nefrología. La enfermedad renal crónica en España [Internet]. Madrid: Sociedad Española de Nefrología; 2018 [Consultado 29 nov 2018]. Disponible en: https://www.senefro.org/ contents/webstructure/comunicacion/SEN_dossier_ Enfermedad_Renal_Cro.pdf.

5. Arrieta J. Evaluación económica del tratamiento sustitutivo renal (hemodiálisis, diálisis peritoneal y trasplante) en España. Nefrología (Madr.). 2010;1(Supl Ext 1):S37-47.

6. Prieto $M$, Selgas R, Pérez-Fontán M, Pérez Contreras FJ, Remón C, Julián JC; en representación del Grupo de Apoyo al Desarrollo de la Diálisis Peritoneal en España (GADDPE). Tratamiento sustitutivo renal: La tormenta perfecta. eSEDISA. 2018;2:1-19.

7. Soriano Cabrera S. Definición y Clasificación de los Estadios de la Enfermedad Renal Crónica. Prevalencia. Claves para el Diagnóstico Precoz. Factores de Riesgo de Enfermedad Renal Crónica. Nefrología. 2004;24(S6):S27-34.

8. Sáenz-Martínez S, Pérez-López F, Martí-García C. Conocimiento sobre la enfermedad renal crónica en la población universitaria de Málaga. Enferm Nefrol. 2019;22(2):186-93.

9. San Juan Miguel Sanz MI, Muñoz Pilar S. Enfermería Nefrológica: de la formación básica a la formación especialista. Rev Soc Esp Enferm Nefrol. 2012;15(3):170-5.

10. Sociedad Española de Nefrología. Promoción del conocimiento en diálisis peritoneal-grupo de apoyo al desarrollo de la diálisis peritoneal [internet]. Santander: Sociedad Española de Nefrología; 2018 [Consultado 29 nov 2018]. Disponible en: http:// www.senefro.org $/$ modules.php? name $=$ grupos\&d $o p=v i e w$-group \&idgroup=4083\&idgroupcontent $=$.

11. Martín Espejo JL, Cirera Segura F. Los conocimientos de Enfermería como Instrumento para el Fomento de la Diálisis Peritoneal. Rev Soc Esp Enferm Nefrol. 2011;14(1):7-13.

12. Carrero JJ, Hecking M, Chesnaye NC, Jager KJ. Sex and gender disparities in the epidemiology and outcomes of chronic kidney disease. Nat Rev Nephrol. 2018;14(3):151-64. 
13. Wright JA, Wallston KA, Elasy TA, Ikizler TA, Cavanaugh KL. Development and Results of a Kidney Disease Knowledge Survey Given to Patients With CKD. Am J Kidney Dis. 2011;57(3):387-5.

14. Gorostidi M, Sánchez-Martínez M, Ruilope LM, Graciano A, De la Cruz JJ, Santamaría R, et al. Prevalencia de enfermedad renal crónica en España: impacto de la acumulación de factores de riesgo cardiovascular. Nefrología 2018;38(6):606-15.

15. Choi B, Granero R, Pak A. Catálogo de sesgos o errores en cuestionarios sobre salud. Rev Costarr Salud Pública. 2010;19(2):106.

Este artículo se distribuye bajo una Licencia Creative Commons Atribución-NoComercial 4.0 Internacional. https://creativecommons.org/licenses/by-nc/4.0/

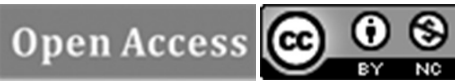

